\title{
STRONGLY CLOSED BOUNDED BOOLEAN ALGEBRAS OF PROJECTIONS
}

\author{
by T. A. GILLESPIE
}

(Received 28 July, 1979)

It is known that every complete Boolean algebra of projections on a Banach space $X$ is strongly closed and bounded and that, although the converse of this result fails in general, it is valid if $X$ is weakly sequentially complete [1, XVII. 3, pp. 2194-2201]. In the present note it is shown that this converse is in fact valid precisely when $X$ contains no subspace isomorphic to the sequence space $c_{0}$. More explicitly, the following two results are proved. In both, $X$ may be a real or complex space, but $c_{0}$ will consist of the null sequences in the underlying scalar field.

THEOREM 1. Let $X$ be a Banach space containing no subspace isomorphic to $c_{0}$. Then every strongly closed bounded Boolean algebra of projections on $X$ is complete.

Theorem 2. Let $X$ be a Banach space containing a subspace isomorphic to $c_{0}$. Then there exists a strongly closed bounded Boolean algebra of projections on $X$ which is not complete.

Since a Banach space containing a subspace isomorphic to $c_{0}$ cannot be weakly sequentially complete, Theorem 1 generalizes the converse result referred to above. Furthermore, this generalization is genuine in the sense that there exist Banach spaces which are not weakly sequentially complete and which contain no subspace isomorphic to $c_{0}$. An example of such a space is the Banach space $J$ of R. C. James [2, p. 25] (taking complex scalars if a complex space is desired). As observed in [2], $J$ does not contain a subspace isomorphic to $c_{0}$ since it has a separable second dual. On the other hand, $J$ is not weakly sequentially complete. To see this, let $\left\{e_{n}\right\}$ be the natural basis of $J$ as in [2, p. 25]. Using the fact that $\left\{e_{n}\right\}$ is a shrinking basis, it is readily verified that the sequence $\left\{e_{1}+\cdots+e_{n}\right\}$ of unit vectors in $J$ is weakly Cauchy but not weakly convergent. In the context of this example, it should be mentioned that there are classes of Banach spaces for which weak sequential completeness is equivalent to the property of possessing no subspace isomorphic to $c_{0}$. (See [3] for more details and further references.)

Proof of Theorem 1. Let $\mathbf{B}$ be a strongly closed Boolean algebra of projections on $X$, where $X$ contains no subspace isomorphic to $c_{0}$, and let $M$ be a positive constant such that $\|E\| \leqslant M$ for all $E$ in $\mathbf{B}$. Suppose $\mathbf{B}$ is not complete. Then, as in the proof of [1, XVII. 3.5, p. 2199], there exist $x$ in $X, \varepsilon>0$ and an increasing sequence $\left\{E_{n}\right\}$ in $\mathbf{B}$ such that

$$
\left\|E_{n+1} x-E_{n} x\right\| \geqslant \varepsilon
$$

for all $n$. Given a sequence $\left\{\xi_{n}\right\}$ of scalars, we have

$$
\left\|\sum_{n=j}^{k} \xi_{n}\left(E_{n+1}-E_{n}\right)\right\| \leqslant 4 M \max \left\{\left|\xi_{n}\right|: j \leqslant n \leqslant k\right\}
$$

Glasgow Math. J. 22 (1981) 73-75. 
for all $j, k$ with $j \leqslant k$ [1, XVII. 2.2, p. 2181]. (The constant $4 M$ can be replaced by $2 M$ in the real case.) It follows that, if $\xi_{n} \rightarrow 0$, then

$$
\sum_{n=1}^{\infty} \xi_{n}\left(E_{n+1}-E_{n}\right)
$$

converges in norm. Let

$$
T \xi=\sum_{n=1}^{\infty} \xi_{n}\left(E_{n+1}-E_{n}\right) x
$$

for $\xi=\left\{\xi_{n}\right\}$ in $c_{0}$. Then $T$ is a linear mapping of $c_{0}$ into $X$ satisfying

$$
\|T \xi\| \leqslant 4 M\|x\|\|\xi\|_{\infty} \quad\left(\xi \in c_{0}\right)
$$

Also, given $\xi=\left\{\xi_{n}\right\}$ in $c_{0}$,

$$
\begin{aligned}
\varepsilon\left|\xi_{n}\right| & \leqslant\left\|\xi_{n}\left(E_{n+1}-E_{n}\right) x\right\| \\
& =\left\|\left(E_{n+1}-E_{n}\right) T \xi\right\| \\
& \leqslant M\|T \xi\|
\end{aligned}
$$

for all $n$. Hence

$$
\|\xi\|_{\infty} \leqslant M \varepsilon^{-1}\|T \xi\| \quad\left(\xi \in c_{0}\right) .
$$

Thus the range of $T$ is a subspace of $X$ isomorphic to $c_{0}$. This gives the required contradiction and completes the proof.

Proof of Theorem 2. Let $X$ contain a subspace $Y$ isomorphic to $c_{0}$. then $Y$ is also isomorphic to the space $c$ of all convergent sequences. Identify $Y$ with $c$, so that $c$ is actually contained in $X$ algebraically as a subspace, with the usual supremum norm $\|\cdot\|_{\infty}$ equivalent to the norm $\|\cdot\|$ inherited from $X$.

Let $\mathbf{N}$ denote the set of positive integers and, for $k \in \mathbf{N}$, let $e_{k}$ denote the element of $c$ with 1 in the $k$ th place and 0 elsewhere. Also, let $\phi_{k}$ denote the coordinate functional

$$
\phi_{k}\left(\left\{x_{n}\right\}\right)=x_{k}
$$

on $c$ and let $\psi_{k}$ be a norm-preserving linear extension of $\phi_{k}$ from $c$, with norm $\|\cdot\|$, to $X$. Note that $\left\{\psi_{k}\right\}$ is then a bounded sequence in the dual space of $X$.

Let $\mathscr{F}$ denote the set of finite subsets of $\mathbf{N}$. Given a non-empty element $\sigma$ of $\mathscr{F}$, define $E_{\sigma}: X \rightarrow X$ by

$$
E_{\sigma} x=\sum_{k \in \sigma} \psi_{k}(x) e_{k} \quad(x \in X)
$$

Notice that the range of such a mapping $E_{\sigma}$ is contained in $c$ and its restriction to $c$ is the natural coordinate projection associated with $\sigma$. Let $E_{\sigma}=0$ if $\sigma$ is the empty set. It is readily verified that

$$
\mathbf{B}=\left\{E_{\sigma}: \sigma \in \mathscr{F}\right\} \cup\left\{I-E_{\sigma}: \sigma \in \mathscr{F}\right\}
$$


is a Boolean algebra of projections on $X$. Given a non-empty $\sigma$ in $\mathscr{F}$,

$$
\begin{aligned}
\left\|E_{\sigma} x\right\|_{\infty} & =\max \left\{\left|\psi_{k}(x)\right|: k \in \sigma\right\} \\
& \leqslant\left\{\sup _{k \in \mathbb{N}}\left\|\psi_{k}\right\|\right\}\|x\|
\end{aligned}
$$

for all $x$ in $X$, and this implies that $\mathbf{B}$ is bounded. Noting that the sequence $\left\{E_{\sigma(n)} e\right\}$ does not converge in $c$, where $e$ is the element $(1,1, \ldots)$ of $c$ and

$$
\sigma(n)=\{2,4, \ldots, 2 n\}
$$

for $n \in \mathbf{N}$, it is immediate from [1, XVII. 3.4, p. 2197] that $\mathbf{B}$ is not complete.

To complete the proof, we show that $\mathbf{B}$ is strongly closed. Let $E$ belong to the closure of $\mathbf{B}$ in the strong operator topology. Then there is a sequence $\{\tau(n)\}$ in $\mathscr{F}$ such that

$$
E_{\tau(n)} e \rightarrow E e \text { or }\left(I-E_{\tau(n)}\right) e \rightarrow E e .
$$

In both cases, $\left\{E_{\tau(n)} e\right\}$ is convergent and hence there exists $m \in \mathbf{N}$ such that $\tau(n)=\tau(m)$ for $n \geqslant m$. Thus

$$
E e=E_{\tau(m)} e \text { or } E e=\left(I-E_{\tau(m)}\right) e .
$$

Suppose firstly that $E e=E_{\tau(m)} e$ and fix $x$ in $X$. There is a sequence $\{\delta(n)\}$ in $\mathscr{F}$ such that either

$$
E_{\delta(n)} x \rightarrow E x \text { and } E_{\delta(n)} e \rightarrow E e
$$

or

(b)

$$
\left(I-E_{\delta(n)}\right) x \rightarrow E x \quad \text { and } \quad\left(I-E_{\delta(n)}\right) e \rightarrow E e .
$$

As before, $\left\{E_{\delta(n)} e\right\}$ is convergent in both cases, implying that $\delta(n)$ is eventually constant. Since $E e=E_{\tau(m)} e$, case (b) cannot occur and the convergence of $E_{\delta(n)} e$ to $E_{\tau(m)} e$ in (a) implies that $\delta(n)=\tau(m)$ for all large enough $n$. Hence $E x=E_{\tau(m)} x$ and, since $x$ is arbitrary, this shows that $E=E_{\tau(m)}$. A similar argument gives that, if $E e=\left(I-E_{\tau(m)}\right) e$, then $E=I-E_{\tau(m)}$. Thus $E$ belongs to $\mathbf{B}$ and so $\mathbf{B}$ is strongly closed as required.

\section{REFERENCES}

1. N. Dunford and J. T. Schwartz, Linear Operators, Part III: Spectral Operators (WileyInterscience, 1971).

2. J. Lindenstrauss and L. Tzafriri, Classical Banach Spaces I (Springer-Verlag, 1977).

3. L. Tzafriri, Reflexivity in Banach lattices and their subspaces, J. Functional Analysis $\mathbf{1 0}$ (1972), 1-18.

Department of Mathematics

James Clerk Maxwell Building

THE KING's BUILDINGS

EDINBURGH EH9 3JZ 